\title{
Polyethylene glycol vs sodium picosulfate/magnesium citrate for colonoscopy preparation
}

Authors

Institutions
Kristian Leitao', Tore Grimstad', Michael Bretthauer ${ }^{2}$, Øyvind Holme ${ }^{3,5}$, Vemund Paulsen² ${ }^{2}$ Lars Karlsen', Kjetil Isaksen ${ }^{1}$, Milada Cvancarova ${ }^{4}$, Lars Aabakken ${ }^{2}$

Institutions are listed at the end of article. submitted 19. May 2014 accepted after revision 6. June 2014

\section{Bibliography}

DOI http://dx.doi.org/

10.1055/s-0034-1377520

Published online: 24.10.2014

Endoscopy International Open 2014; 02: E230-E234

(c) Georg Thieme Verlag KG Stuttgart · New York

E-ISSN 2196-9736

\section{Corresponding author}

\section{Dr. Kristian Leitao}

Stavanger University Hospital Stavanger

Norway

Phone: +47-51-51-80-53

Norway

leko@sus.no
Background and study aims: Polyethylene glycolbased electrolyte solutions (PEG-ELS) and the combination of sodium picosulfate/magnesium citrate (SPMC) are commonly used bowel preparation agents. The aim of the present study was to compare the two agents with regard to cleansing efficacy and tolerance among individuals scheduled for outpatient colonoscopy.

Materials and methods: The 368 colonoscopy outpatients at three Norwegian hospitals were randomized to bowel lavage with either PEG-ELS or SPMC. Compliance and patient tolerance were evaluated using a patient questionnaire. Bowel cleansing was evaluated using the Ottawa Bowel Preparation Quality Scale (OBPS), a validated scoring system with scores between 0 (best) and 14 .

$\begin{array}{ll}\text { Abbreviations } \\ \boldsymbol{\nabla} \\ \text { HD } & \text { High definition } \\ \text { NBI } & \text { Narrow band imaging } \\ \text { OBPS } & \text { Ottawa Bowel Preparation Quality Scale } \\ \text { SPMC } & \text { Sodium picosulfate/magnesium citrate }\end{array}$

\section{Introduction}

$\nabla$

Effective bowel cleansing is a prerequisite for optimal investigation of the colon during colonoscopy. Inadequate bowel cleansing decreases adenoma detection rate and cecal intubation rate. It also limits the utility of high definition endoscopy (HD) and narrow band imaging (NBI), and increases the level of technical difficulty, time spent and procedural risks [1,2].

The ideal bowel-cleansing preparation not only effectively discharges bowel contents, but also convenient and tolerable for the patient, and without risk of complications. Screening programs for colorectal cancer impose the burden of bowel cleansing and colonoscopy on healthy adults. Getting patients to participate in these
Results: There was no difference in the cleansing quality between the PEG-ELS and SPMC groups (median OBPS 5.0 in both groups). The group that received SPMC reported better overall patient tolerance than the PEG-ELS group (72.6\% vs $59.0 \%$ reporting no or slight discomfort, $P<0.01$ ). Compliance with the recommended total fluid intake $(4 \mathrm{~L})$ was better in the SPMC group than in the PEG-ELS group ( $94.2 \%$ vs $81.2 \%$ respectively, $P<0.01)$; moreover, the polyp detection rate was superior ( $34.3 \%$ vs $23.3 \%, P=0.02$ ).

Conclusion: PEG-ELS and SPMC are equally effective in cleansing efficacy, but SPMC was better tolerated by patients and resulted in superior patient compliance and polyp detection rate.

Clinical trial registration: NCT01624454

programs is challenging $[3,4]$ and the overall experience (including the cleansing procedure) influences the patient's willingness to undergo screening colonoscopy or repeat procedures [5]. In one survey, $77 \%$ of patients rated the cleansing procedure as the most difficult part of the procedure [6].

The three most commonly used categories of bowel-cleansing agents are polyethylene glycolbased electrolyte solutions (PEG-ELS), osmotically active agents, and stimulant laxatives. Some preparations and schemes combine these mechanisms.

PEG-ELS contains macrogol, a polymer in an osmotically balanced dilute electrolyte solution. This preparation is ingested in a high volume, typically $2-4 \mathrm{~L}$, and flushes the bowel contents without a fluid shift across the bowel wall. The volume itself induces peristaltic activity. PEG-ELS is often combined with motility stimulants. It is considered safe, even for patients with electrolyte imbalances, or those with liver, heart, or renal failure $[7,8]$. However, patient tolerance has been a concern, and several trials have found PEG-ELS 
is not tolerated by patients as well as osmotically active agents [8].

Osmotically active agents are small volume preparations, which draw fluids into the bowel lumen, thus achieving the same volume effect as PEG-ELS. These substances are considered more tolerable with regard to taste and volume, but carry the risk of electrolyte disturbances, volume depletion, and aggravation of renal failure [9]. Hence, it is essential that these preparations are taken with sufficient amounts of clear liquids to maintain fluid balance, and should be used only by patients with low risk of these side effects. Sodium phosphate has proven to be more effective with regard to bowel cleansing than PEG-ELS in several trials. However, it is associated with electrolyte disturbances such as hypophosphatemia, hypocalcemia, hypokalemia, and hyper- or hyponatremia, and has been reported to induce acute phosphate nephropathy with renal failure $[8,10,11]$. Guidelines generally advise against the routine use of sodium phosphate [12].

Motility stimulants increase the smooth muscle activity within the bowel wall and induce hyperperistalsis. These are mostly used in combination with other agents for bowel cleansing.

Sodium picosulfate is a stimulant cathartic that colonic bacteria metabolize to its active compound diphenol, which increases colonic motility. Magnesium citrate is an osmotically active laxative, which draws fluid into the gut lumen and partly stimulates peristalsis by release of cholecystokinin. Unlike sodium phosphate, products combining sodium picosulfate and magnesium citrate (SPMC) are usually not associated with significant electrolyte disturbances [13-15]. The most important concern is the risk of hypermagnesemia in patients with renal failure, as magnesium is eliminated solely by the kidney [15].

The most efficacious and well-tolerated bowel preparation has not yet been found; there is a considerable heterogeneity in results from previous trials [8]. The aim of the present trial was to compare PEG-ELS and SPMC, the two preferred bowel preparations according to recent European Society of Gastrointestinal Endoscopy guidelines [12], with regard to their cleansing efficacy, patient tolerance, and patient compliance among symptomatic and surveillance outpatients undergoing elective colonoscopy. In addition, endoscopic and clinical outcomes were evaluated.

\section{Materials and method \\ $\nabla$}

\section{Patients and study design}

In this randomized investigator-blinded trial, consecutive patients aged 18 to 80 years scheduled for outpatient colonoscopy at three Norwegian hospitals between March and October 2012 were considered eligible. Exclusion criteria were active colitis, suspicion of renal insufficiency or ileus/subileus, previous colonic surgery, pregnancy, inability to adhere to cleansing instructions, and inability to consent.

Eligible patients were randomized 1:1 to either PEG-ELS or SPMC using block randomization with blocks of 10 by the endoscopyunit secretary. All eligible patients received a letter of invitation to participate in the study together with their appointment confirmation for colonoscopy, and a description of the bowel-cleansing procedure according to their assigned study group. Participating patients also received a questionnaire concerning their tolerance to and the efficacy of the cleansing procedure, to be filled out during the course of the cleansing process, and completed at the latest before the start of the colonoscopy. This questionnaire was collected by the endoscopy assistant/nurse on arrival at the outpatient clinic.

\section{Endpoints}

The primary endpoint was the quality of bowel cleansing. Secondary endpoints were patient tolerance, and patient compliance, polyp detection rate, and cecal intubation time and rate.

\section{Cleansing procedure}

For both the PEG-ELS and SPMC preparations, patients were advised to avoid iron preparations and food with seeds during the week before the colonoscopy. After 12 noon the day before the colonoscopy, only clear liquids were recommended. Both preparations were administered as a split-dose.

The patients randomized to PEG-ELS (Endofalk, Falk Pharma, Freiburg, Germany) were instructed to mix six sachets $(52.5 \mathrm{~g}$ Macrogol plus electrolytes [ $1.4 \mathrm{~g}$ sodium chloride, $0.185 \mathrm{~g}$ potassium chloride, $0.715 \mathrm{~g}$ sodium bicarbonate] per sachet) of Endofalk powder with $3 \mathrm{~L}$ of water; $2 \mathrm{~L}$ were ingested at $6 \mathrm{p} . \mathrm{m}$. the day before the colonoscopy, and $1 \mathrm{~L}$ the next morning together with at least $1 \mathrm{~L}$ of clear liquids.

The patients randomized to SPMC (CitraFleet, C.B. Fleet Company, Virginia, United States) were instructed to mix two sachets (10 $\mathrm{mg}$ of sodium picosulfate, $3.5 \mathrm{~g}$ magnesium oxide and $10.97 \mathrm{~g}$ citric acid per sachet) of CitraFleet powder each with $150 \mathrm{~mL}$ of water. The first $150 \mathrm{~mL}$ were ingested at 1800 the day before the colonoscopy, followed by at least $2 \mathrm{~L}$ of clear liquids. The remaining $150 \mathrm{~mL}$ were ingested the next morning, followed by at least $2 \mathrm{~L}$ of clear liquids.

For both cleansing agents, the recommended total volume of fluid ingested was $4 \mathrm{~L}$, and patients were informed that they were allowed to drink additional clear liquids at will until the start of the procedure. The morning dose was taken at 0500 for those scheduled for colonoscopy before 1200 , and at 7 a.m. for those scheduled for colonoscopy after 1200.

\section{Patient assessment of cleansing procedure}

Patients answered a questionnaire designed by the study group, during the colon cleansing, before start of the colonoscopy. The start and end times for the bowel preparation were recorded as was the total volume of cleansing agent consumed. The perception of discomfort experienced (nausea/vomiting, bloating, incontinence, abdominal pain/cramps, overall discomfort) was rated on a 5-point scale as follows: 0 , no discomfort; 1 , slight discomfort; 2 , moderate discomfort; 3 , significant discomfort; 4 , severe discomfort.

\section{Endoscopic procedure and cleansing efficacy assessment} The colonoscopy was performed according to the normal standard of care, using instruments, sedation, and techniques at the discretion of the endoscopist. Colonoscopes were CF-H160, CFH180 and CF-H190 (Olympus, Tokyo, Japan). The endoscopist was blinded with regard to the bowel cleansing regimen used. All endoscopists were experienced having performed at least 500 colonoscopies.

The bowel-cleansing quality was evaluated using the Ottawa Bowel Preparation Quality Scale (OBPS), a validated scoring system with scores between 0 and 14, where 0 is the best score [16]. The score comprises a sub score $0-4$ for each colon segment: right, transverse and rectosigmoid colon. In addition, a score $0-2$ is added to indicate the total amount of luminal fluid. 
Before initiating the trial, a training video for the OBPS was distributed to the investigators, and a poster with photos demonstrating the different Ottawa scores was displayed in each colonoscopy room to ensure correct scoring.

\section{Statistics}

The study was designed as a randomized controlled investigatorblinded trial where a difference of one in Ottawa score between the cleansing regimens was considered clinically relevant. To determine the sample size, we estimated the distribution parameters from the first 200 patients included (mean and SD) without revealing their group assignments. Based on an estimated difference of 1 , an assumed equal SD of 2.7 for both groups, a power of $90 \%$, and an alpha value of $5 \%, 155$ patients were required in each group. Differences between groups for categorical variables were assessed with chi-squared tests. Continuous variables were not normally distributed. The Mann-Whitney test was therefore used and data were described with median and range. $P$ values $<$ 0.05 were considered statistically significant. All tests were twosided. For all statistical analyses, SPSS version 16.0 was used. Corrections for multiple analyses were not performed.

\section{Ethics}

The study was performed in accordance with the Declaration of Helsinki. Patients received written information together with the summons letter for colonoscopy, which was brought to the procedure together with a signed consent form. Both colon cleansing agents are well accepted standard procedures in Norway and are used interchangeably. The study was waived for assessment by the Regional Ethics committee and authorized by the data protection officer at the participating hospitals. The study was not industry sponsored, and no author has a conflict of interest.

\section{Results \\ $\nabla$}

\section{Patient characteristics}

A total of 368 patients were included in the trial. 189 patients were randomized to the PEG-ELS group, and 179 patients to the SPMC group. Demographics and clinical characteristics were similar in both arms ( Table 1). Visible blood, diarrhea and polyp surveillance were the most common indications ( $\bullet$ Table 2 ).

\section{Bowel-cleansing efficacy}

The quality of the bowel cleansing as assessed by an endoscopist revealed a median Ottawa score of 5.0 (range $0-14$ ) in the PEGELS group and 5.0 (range $0-13$ ) in the SPMC group, resulting in no significant difference between the two groups with regard to overall cleansing effect. There were also no differences between the agents in cleansing effect on the individual colon segments (๑ Table 3).

\section{Patient tolerance to bowel cleansing}

Nausea and vomiting were significantly more frequent among participants in the PEG-ELS group $(P=0.001)$, and the overall experience was significantly worse $(P=0.007)$ compared to SPMC ( Table 3). No adverse reactions were observed beyond those recorded in the patient questionnaire.
Table 1 Characteristics and indications of patients for colonoscopy who were randomized into polyethylene glycol electrolyte solution (PEG-ELS) or sodium picosulfate/magnesium citrate (SPMC) treatment groups.

\begin{tabular}{|lll|}
\hline & PEG-ELS & SPMC \\
\hline Participants & 189 & 179 \\
\hline Sex (M/F) & $89 / 100$ & $89 / 90$ \\
\hline Age, median (range), years & $61(18-85)$ & $64(19-84)$ \\
\hline
\end{tabular}

Table 2 Main indications for colonoscopy among patients who received polyethylene glycol electrolyte solution (PEG-ELS) $(n=165)$ or sodium picosulfate/magnesium citrate (SPMC) $(n=163)$ as a bowel-cleansing agent.

\begin{tabular}{|lcc|}
\hline & PEG-ELS & SPMC \\
\hline Symptom & $\mathrm{n}(\%)$ & $\mathrm{n}(\%)$ \\
\hline Visible blood & $32(16.9)$ & $35(19.6)$ \\
\hline Diarrhea & $23(12.2)$ & $27(15.1)$ \\
\hline Polyp surveillance & $26(13.8)$ & $25(14.0)$ \\
\hline Surveillance & $21(11.1)$ & $21(11.7)$ \\
\hline Abdominal pain & $24(12.7)$ & $19(10.6)$ \\
\hline Occult blood & $1(0.5)$ & $15(8.4)$ \\
\hline CT/MR finding & $7(3.7)$ & $7(3.9)$ \\
\hline Suspected CRC & $4(2.1)$ & $6(3.4)$ \\
\hline Constipation & $5(2.6)$ & $3(1.7)$ \\
\hline Screening & $6(3.2)$ & $2(1.1)$ \\
\hline Diverticulitis & $8(4.2)$ & $1(0.6)$ \\
\hline Altered bowel habits & $5(2.6)$ & $1(0.6)$ \\
\hline Other & $3(1.6)$ & $1(0.6)$ \\
\hline Total & $165(87.3)$ & $163(91.1)$ \\
\hline Missing data & $24(12.7)$ & $16(8.9)$ \\
\hline Total & $189(100.0)$ & $179(100.0)$ \\
\hline
\end{tabular}

Table 3 Ottawa bowel preparation quality scale (OBPS) in different colon segments as registered in 368 patients who ingested polyethylene glycol electrolyte solution (PEG-ELS) or sodium picosulfate/magnesium citrate (SPMC) as a bowel preparation before colonoscopy. The median score (range) is presented.

\begin{tabular}{|llll|}
\hline & $\begin{array}{l}\text { PEG-ELS } \\
\text { N=189 }\end{array}$ & $\begin{array}{l}\text { SPMC } \\
\mathbf{N}=179\end{array}$ & P value $^{1}$ \\
\hline OBPS & $5(0-14)$ & $5(0-13)$ & 0.30 \\
\hline Right colon & $2(0-4)$ & $2(0-4)$ & 0.18 \\
\hline Mid colon & $2(0-4)$ & $2(0-4)$ & 0.98 \\
\hline Rectosigmoid colon & $2(0-4)$ & $2(0-4)$ & 0.29 \\
\hline Total fluid & $1(0-2)$ & $1(0-2)$ & 0.82 \\
\hline
\end{tabular}

${ }^{1}$ Mann Whitney U-test

\section{Patient compliance with bowel-cleansing regimen}

Of the patients who ingested SPMC, 10 patients (5.8\%) did not consume the full prescribed volume of $4 \mathrm{~L}$. In the PEG-ELS group, 34 patients $(18.8 \%)$ were not able to consume the full prescribed volume of $4 \mathrm{~L}(P=0.001)(\checkmark$ Table 4$)$. However, no statistically significant differences in OBPS were observed between compliant and the non-compliant patients who had median scores of 5 and 6 , respectively $(P=0.08)$.

\section{Colonoscopy performance}

Cecal intubation rate was $96 \%$ for both preparations. Median cecal intubation time was $11 \mathrm{~min}$ for PEG-ELS and $8 \mathrm{~min}$ for SPMC, however, the difference was not statistically significant. In one 
Table 4 Patient tolerance of bowel cleansing as assessed by reported side effects after ingestion of polyethylene glycol electrolyte solution (PEG-ELS) or sodium picosulfate (SPMC) in 368 patients before colonoscopy ( $\mathrm{n}(\%))$.

\begin{tabular}{|c|c|c|c|c|c|c|c|}
\hline Side effect & Cleansing agent & No discomfort & Slight & Moderate & Significant & Severe & $\begin{array}{l}P \text { value }^{1} \\
\text { (Total) }\end{array}$ \\
\hline \multirow[t]{2}{*}{ Overall experience } & PEG-ELS & $59(34)$ & $43(25)$ & $36(21)$ & $25(14)$ & $10(6)$ & \multirow[t]{2}{*}{0.007} \\
\hline & SPMC & $73(45)$ & $46(28)$ & $27(16)$ & $13(8)$ & $5(3)$ & \\
\hline \multirow[t]{2}{*}{ Nausea/vomiting } & PEG-ELS & $79(43)$ & $50(27)$ & $26(14)$ & $19(10)$ & $8(4)$ & \multirow[t]{2}{*}{$<0.001$} \\
\hline & SPMC & $108(64)$ & $41(24)$ & $11(7)$ & $5(3)$ & $4(2)$ & \\
\hline \multirow[t]{2}{*}{ Bloating } & PEG-ELS & $100(56)$ & $51(29)$ & $13(7)$ & $13(7)$ & $1(1)$ & \multirow[t]{2}{*}{0.45} \\
\hline & SPMC & $102(60)$ & $44(26)$ & $17(10)$ & $5(3)$ & $2(1)$ & \\
\hline \multirow[t]{2}{*}{ Leakage/incontinence } & PEG-ELS & $130(76)$ & $26(15)$ & $3(2)$ & $8(5)$ & $4(2)$ & \multirow[t]{2}{*}{0.24} \\
\hline & SPMC & $135(82)$ & $13(8)$ & $10(6)$ & $4(2)$ & $3(2)$ & \\
\hline \multirow[t]{2}{*}{ Abdominal pain/cramps } & PEG-ELS & $130(75)$ & $24(14)$ & $12(7)$ & $8(5)$ & 0 & \multirow[t]{2}{*}{0.89} \\
\hline & SPMC & $124(73)$ & $30(18)$ & $8(5)$ & $5(3)$ & $2(1)$ & \\
\hline
\end{tabular}

${ }^{1}$ Mann Whitney U-test

patient in the PEG-ELS group, poor bowel cleansing prevented the approach of the colonoscope to the cecum; this problem did not occur among any of the patients in the SPMC group. The polyp detection rate was significantly higher in patients who ingested SPMC as cleansing agent ( $34.3 \%$ vs $23.3 \%, P=0.02$ ) ( $\bullet$ Table 5).

\section{Discussion}

$\nabla$

The main finding in this study was that split-dose PEG-ELS (EndoFalk) and split-dose SPMC (CitraFleet) were equally effective with regard to bowel cleansing as measured by the OBPS. However, SPMC was tolerated by patients better than PEG-ELS as shown by significantly better patient-reported outcomes for tolerance and nausea/vomiting. In addition, patient compliance was significantly higher in the SPMC group. Furthermore, we observed a higher polyp detection rate among patients who consumed SPMC as compared to PEG-ELS.

Previous trials have shown similar results in terms of cleansing efficacy $[12,17]$. Concerning the overall cleansing quality, a total Ottawa score of 5 or less is regarded as satisfactory, this is, however, based on expert opinion [18].

SPMC was better tolerated than PEG-ELS and led to better patient compliance. One could expect better cleansing quality in the SPMC group, but only a trend toward a worse Ottawa score was observed when comparing all non-compliant with compliant patients. However, the number of non-compliant patients was small and the distinction between compliant vs non-compliant patients was ill defined.

Although no difference in the Ottawa score was detected, the polyp detection rate was higher in the SPMC group.The reason for this finding is not clear. It may be a random finding, but it is probably multifactorial and could be related to withdrawal time, endoscopist experience, patient position, and bowel distension; however, these data were not collected. It is important to recall that the OBPS evaluates bowel preparation before endoscopic cleansing by flushing and suctioning, not taking into account the final result after the endoscopist's efforts to clear colon contents. The Boston scale is probably better adapted to evaluate this particular clinical outcome as it is applied after endoscopic clearing of residual luminal contents [19].

Strengths of this trial include the design of an adequately powered multicenter trial and following a strict protocol. The two currently most attractive bowel preparations were administered
Table 5 Procedural outcomes, polyps detected, and patient compliance among 368 patients who ingested polyethylene glycol electrolyte solution (PEG-ELS) or sodium picosulfate/magnesium citrate (SPMC) as bowel preparation before colonoscopy ( $\mathrm{n}[\%])$.

\begin{tabular}{|c|c|c|c|}
\hline & $\begin{array}{l}\text { PEG-ELS } \\
(n=189)\end{array}$ & $\begin{array}{l}\text { SPMC } \\
(n=179)\end{array}$ & $P$ value \\
\hline $\begin{array}{l}\text { Cecal intubation time, median } \\
\text { (range), min }\end{array}$ & $11(2-69)$ & $8(2-39)$ & $0.47^{1}$ \\
\hline Cecal intubation & $182(96.3)$ & $173(96.6)$ & $0.86^{2}$ \\
\hline Polyp(s) detected ${ }^{3}$ & $44(23.3)$ & $60(34.3)$ & $0.02^{4}$ \\
\hline \multicolumn{4}{|l|}{ Fluid intake ${ }^{4}$} \\
\hline$<4 \mathrm{~L}$ & $34(18.9)$ & $10(5.8)$ & $0.001^{4}$ \\
\hline$\geq 4 \mathrm{~L}$ & $146(81.1)$ & $161(94.2)$ & \\
\hline
\end{tabular}

${ }^{1}$ Mann Whitney U test

${ }^{2}$ Chi-squared test

${ }^{3}$ Missing data for four patients in the SPMC group

${ }^{4}$ Missing data for fluid intake in 17 patients: 9 in the PEG-ELS group and 8 in the SPMC group.

as a split-dose to optimize both efficacy and patient tolerance. This is in accordance with the European Society of Gastrointestinal Endoscopy guidelines.

As for study limitations, there were missing data for some variables among some patients. The questionnaires for both patients and endoscopists were not always fully completed, but we have no reason to believe there were any systematic mistakes that would lead to biased results. The questionnaires assessing patient tolerance and compliance were designed by the study group and have not been previously validated. Polyp histology was not available, hence we cannot report on the adenoma detection rate. In conclusion, PEG-ELS and SPMC were equally efficient with regard to bowel cleansing. Sodium picosulfate/magnesium citrate was better tolerated by patients and yielded better patient compliance and a superior polyp detection rate.

\section{Competing interests: None}

\section{Institutions}

${ }^{1}$ Division of Gastroenterology, Department of Medicine, Stavanger University Hospital, Stavanger, Norway

2 Department of Transplantation Medicine, Oslo University Hospital - Rikshospitalet, Oslo, Norway

3 Department of Medicine, Sorlandet Hospital, Kristiansand, Norway

${ }^{4}$ Department of Biostatistics, University of Oslo, Norway

${ }^{5}$ Insitute for Health and Society, University of Oslo, Norway 


\section{References}

1 Canard JM, Debette-Gratien M, Dumas $R$ et al. A prospective national study on colonoscopy and sigmoidoscopy in 2000 in France. Gastroenterol Clin Biol 2005; 29: 17-22

2 Froehlich F, Wietlisbach $V$, Gonvers JJ et al. Impact of colonic cleansing on quality and diagnostic yield of colonoscopy: the European Panel of Appropriateness of Gastrointestinal Endoscopy European multicenter study. Gastrointest Endosc 2005; 61: 378 - 384

3 Quintero E, Castells A, Bujanda $L$ et al. Colonoscopy vs fecal immunochemical testing in colorectal-cancer screening. NEJM 2012; 366: 697-706

4 Segnan N, Senore C, Andreoni B et al. Comparing attendance and detection rate of colonoscopy with sigmoidoscopy and FIT for colorectal cancer screening. Gastroenterology 2007; 132: 2304-2312

5 Senore C, Ederle A, Fantin A et al. Acceptability and side effects of colonoscopy and sigmoidoscopy in a screening setting. J Med Screen 2011; 18: $128-134$

6 Ko CW, Riffle S, Shapiro JA et al. Incidence of minor complications and time lost from normal activities after screening or surveillance colonoscopy. Gastrointest Endosc 2007; 65: 648

7 Marschall HU, Bartels F. Life-threatening complications of nasogastric administration of polyethylene glycol-electrolyte solutions (Golytely) for bowel cleansing. Gastrointest Endosc 1998; 47: 408-410

8 Barkun A, Chiba N, Enns R et al. Commonly used preparations for colonoscopy: efficacy, tolerability, and safety-a Canadian Association of Gastroenterology position paper. Can J Gastroenterol 2006; 20: 699710

9 Lichtenstein G. Bowel preparations for colonoscopy: a review. Am J Health Syst Pharm 2009; 66: 27-37

10 Clarkston WK, Tsen TN, Dies DF et al. Oral sodium phosphate vs. sulfatefree polyethylene glycol electrolyte lavage solution in outpatient prep- aration for colonoscopy: a prospective comparison. Gastrointest Endosc 1996; 43: $42-48$

11 Casais MN, Rosa-Diez G, Perez S et al. Hyperphosphatemia after sodium phosphate laxatives in low risk patients: prospective study. World J Gastroenterol 2009; 15: 5960 - 5965

12 Hassan C, Bretthauer M, Kaminski MF et al. Bowel preparation for colonoscopy: European Society of Gastrointestinal Endoscopy (ESGE) guideline. Endoscopy 2013; 45: 142 - 150

13 Rahman A, Vanner SJ, Baranchuk A et al. Serial monitoring of the physiological effects of the standard Pico-Salax(R) regimen for colon cleansing in healthy volunteers. Can J Gastroenterol 2012; 26: 424 428

14 Lawrance JA, Massoud TF, Creasy TS et al. Colonic preparation with Picolax: patient tolerance and approaches to fluid replacement. Clin Radiol 1994; 49: 35 - 37

15 Hookey LC, Vanner S. A review of current issues underlying colon cleansing before colonoscopy. Can J Gastroenterol 2007; 21: 105-111

16 Rostom A, Jolicoeur E. Validation of a new scale for the assessment of bowel preparation quality. Gastrointest Endosc 2004; 59: 482 - 486

17 Voiosu T, Ratiu I, Voiosu A et al. Time for individualized colonoscopy bowel-prep regimens? A randomized controlled trial comparing sodium picosulfate and magnesium citrate vs. 4-liter split-dose polyethylene glycol J Gastrointestin Liver Dis 2013; 22: 129-134

18 Seo EH, Kim TO, Park MJ et al. Optimal preparation-to-colonoscopy interval in split-dose PEG bowel preparation determines satisfactory bowel preparation quality: an observational prospective study. Gastrointest Endosc 2012; 75: 583-590

19 Calderwood AH, Jacobson BC. Comprehensive validation of the Boston Bowel Preparation Scale. Gastrointest Endosc 2010; 72: 686 - 692 